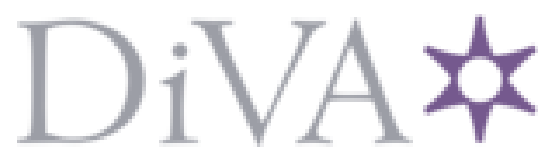

http://www.diva-portal.org

This is the published version of a paper published in Applied Physics Letters.

Citation for the original published paper (version of record):

Islam, M. (2014)

Stable magnetic order and charge induced rotation of magnetizationin nano-clusters.

Applied Physics Letters, 105(152409)

http://dx.doi.org/10.1063/1.4898670

Access to the published version may require subscription.

N.B. When citing this work, cite the original published paper.

Permanent link to this version:

http://urn.kb.se/resolve?urn=urn:nbn:se:lnu:diva-44555 


\section{A|P I ApDied Physics Letters}

\section{Stable magnetic order and charge induced rotation of magnetization in nano-clusters}

M. Fhokrul Islam and Shiv N. Khanna

Citation: Applied Physics Letters 105, 152409 (2014); doi: 10.1063/1.4898670

View online: http://dx.doi.org/10.1063/1.4898670

View Table of Contents: http://scitation.aip.org/content/aip/journal/apl/105/15?ver=pdfcov

Published by the AIP Publishing

\section{Articles you may be interested in}

Effects of dimensionality and spatial distribution on the magnetic relaxation of interacting ferromagnetic nanoclusters: A Monte Carlo study

J. Appl. Phys. 115, 173906 (2014); 10.1063/1.4873298

Magnetization analysis of oriented chains of hexagonal cobalt nanoplates

J. Appl. Phys. 115, 17B521 (2014); 10.1063/1.4867345

Size-dependence of magneto-electronic coupling in Co nanoparticles

J. Appl. Phys. 113, 223703 (2013); 10.1063/1.4810853

Nano-particle magnetism with a dispersion of particle sizes

J. Appl. Phys. 112, 103915 (2012); 10.1063/1.4766817

Demixing in cobalt clusters embedded in a carbon matrix evidenced by magnetic measurements

J. Appl. Phys. 110, 063904 (2011); 10.1063/1.3638035

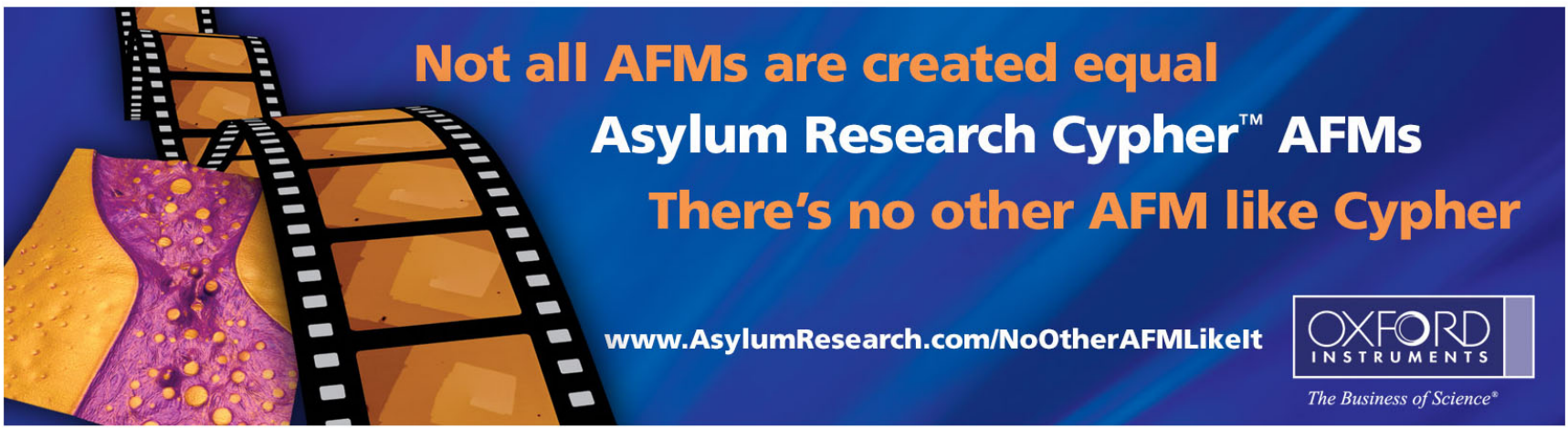




\title{
Stable magnetic order and charge induced rotation of magnetization in nano-clusters
}

\author{
M. Fhokrul Islam and Shiv N. Khanna \\ Department of Physics, Virginia Commonwealth University, Richmond, Virginia 23284-2000, USA
}

(Received 5 August 2014; accepted 5 October 2014; published online 16 October 2014)

\begin{abstract}
Efficient control of magnetic anisotropy and the orientation of magnetization are of central importance for the application of nanoparticles in spintronics. Conventionally, magnetization is controlled directly by an external magnetic field or by an electric field via spin-orbit coupling. Here, we demonstrate a different approach to control magnetization in small clusters. We first show that the low magnetic anisotropy of a $\mathrm{Co}_{5}$ cluster can be substantially enhanced by attaching benzene molecules due to the mixing between $\mathrm{p}$ states of $\mathrm{C}$ and the d states of Co sites. We then show that the direction of magnetization vector of $\mathrm{Co}_{5}$ sandwiched between two benzene molecules rotates by $90^{\circ}$ when an electron is added or removed from the system. An experimental set up to realize such effect is also suggested. (C) 2014 AIP Publishing LLC.

[http://dx.doi.org/10.1063/1.4898670]
\end{abstract}

The realization of spin based devices ${ }^{1}$ is currently one of the most aspiring goal of nano-scale research. Since magnetic nano-structures are the building blocks for the design of spintronic devices, recent advances in nano-fabrication and development of sophisticated experimental techniques ${ }^{2-5}$ not only enhanced our understanding of underlying physics at nanoscale but also have taken us a step closer to developing usable devices. ${ }^{6-9}$ Since electronic spin is central to any spintronic device, it is imperative that the spin states of the system remain stable under thermal fluctuation. Since magnetic anisotropy energy (MAE) can provide such stability, significant efforts have been made to synthesize systems with large MAE, both by synthesizing new magnetic materials ${ }^{10}$ or by depositing nano-structures on substrates. ${ }^{11-13}$ Apart from stabilizing magnetic properties, efficient manipulation of magnetization is also crucial for spintronics application. At nano-scale level, manipulating magnetic states with magnetic field requires one to focus the field at very small spatial and temporal scale, which is very difficult to achieve. In recent years, however, methods for controlling magnetization with an electric field have been developed where magnetic states of a system with strong spin-orbit coupling can be manipulated by modifying orbital distribution by the field, ${ }^{14-17}$ which is more efficient as strong electric field can be easily created at nano-scale level by scanning tunneling microscope (STM) tip.

While most of the previous studies in spintronics are based on semiconductors, ${ }^{8}$ one of the novel classes of systems that have attracted recent attention is one-dimensional organometallic molecular magnets. ${ }^{18}$ In particular, using the gas phase techniques, it is possible to synthesize molecular wires composed of metallic clusters sandwiched between organic molecules. ${ }^{19}$ These systems are appealing as numerous experiments have shown that the magnetic moments in small metallic clusters can be around 30\% more than that in bulk. $^{20-25}$ The reduction in size, however, reduces magnetic anisotropy that leads to fluctuation of orientation of magnetization preventing any application as memory or spintronic devices. If the organic linker could stabilize the orientation, and since it is possible to stabilize multiple decker complexes, it could provide a novel class of one-dimentional systems with stable magnetic order for potential applications.

In this work, we have studied electronic and magnetic properties of $\mathrm{Co}_{5}$ cluster and a simple method to enhance the MAE by sandwiching the cluster between two benzene rings. We show that MAE enhances by a factor of 2.5 when $\mathrm{Co}_{5}$ cluster is sandwiched between two benzene. We also propose a method to rotate magnetization vector simply by adding or by removing a charge from the system. Unlike conventional methods, this approach does not require any external field, magnetic or electric, to rotate magnetization. Microscopic origin of magnetization rotation is also discussed. To study the effects of environment, we have attached the cluster with gold $(\mathrm{Au})$ leads and have investigated its influence on electronic and magnetic properties of the system.

The theoretical studies are carried out using density functional theory (DFT) as implemented in NRLMOL package, ${ }^{26,27}$ which uses Gaussian type orbital as basis. The exchange correlation effects are included using generalized gradient approximation (GGA) as proposed by Perdew et $a l .{ }^{28}$ Previous studies have indicated that this approach can lead to quantitatively accurate results in molecular magnets composed of magnetic clusters linked by organic linkers. For example, our earlier studies of $\mathrm{Mn}_{12} \mathrm{O}_{12}$-acetate lead to a theoretical MAE of $56 \mathrm{~K}$ compared to the experimental value of $55.6 \mathrm{~K}^{29}$ All calculations are performed at an all electron level except for the calculations with gold $(\mathrm{Au})$ lead where pseudo potential is used for $\mathrm{Au}$ atoms. For each case, clusters are relaxed until the forces dropped to about 0.001 Hartree/bohr. Magnetic anisotropy energy (MAE) is calculated by exact diagonalization. In this approach, change in the ground state energies for different magnetization directions are calculated by diagonalizing spin-orbit Hamiltonian in the basis of all Kohn-Sham (KS) orbitals within a given energy window, typically about $50 \mathrm{eV}$ around the Fermi level. MAE is then the difference between the largest and the smallest total energies. While exact diagonalization method is more accurate for MAE calculation, perturbative approach 
is very useful for analyzing the results as we can identify different contribution to MAE in a physically transparent way. Therefore, we have also calculated MAE using 2nd order perturbation theory. The spin-orbit Hamiltonian can be expressed as

$$
\begin{aligned}
H_{S O} & =\lambda \mathbf{L} \cdot \mathbf{S}, \\
& =\lambda\left[L_{\zeta} S_{\zeta}+\frac{1}{2}\left(L_{+} S_{-}+L_{-} S_{+}\right)\right],
\end{aligned}
$$

where, $\lambda$ is the spin-orbit coupling constant, $L_{ \pm}$and $S_{ \pm}$are the ladder operators for orbital and spin states, respectively. The change in the groundstate (GS) energy due to spin-orbit coupling $^{30}$ (SOC) can be expressed using single particle spin-orbital as (Perterbative method in NRLMOL is implemented slightly different but equivalent way, details of which can be found in Ref. 29)

$$
\delta E=-\lambda^{2}[a(\uparrow, \uparrow)+a(\downarrow, \downarrow)-a(\uparrow, \downarrow)-a(\downarrow, \uparrow)],
$$

where

$$
a\left(\sigma, \sigma^{\prime}\right)=\sum_{k k^{\prime}} \frac{\left\langle\phi_{k}, \sigma|\mathbf{L} \cdot \mathbf{S}| \phi_{k}^{\prime}, \sigma^{\prime}\right\rangle\left\langle\phi_{k^{\prime}}, \sigma^{\prime}|\mathbf{L} \cdot \mathbf{S}| \phi_{k}, \sigma\right\rangle}{\epsilon_{k}-\epsilon_{k^{\prime}}} .
$$

Here, $k$ and $k^{\prime}$ represent occupied and unoccupied states, respectively, and $\phi_{k}^{\prime}$ s are Kohn-Sham orbitals. Comparison of MAE obtained from the two approaches can be a useful guide for understanding electronic structure of a cluster. An agreement between exact diagonalization and perturbative calculations indicates that the 2 nd order correction is the main contribution to GS energy. We note from Eq. (3) that the contribution of spin-orbit energy to the GS comes from both spin conserving and spin-flip terms.

First, we discuss the electronic structure of the $\mathrm{Co}_{5}$ cluster. The free $\mathrm{Co}_{5}$ cluster $^{23}$ has $\mathrm{D}_{3 h}$ symmetry with three atoms forming a triangular plane, and the other two atoms are symmetrically placed above and below the plane and on the $\mathrm{C}_{3}$ axis. Since atomic Co has $3 d^{7} 4 s^{2}$ configuration with a spin multiplicity of 4 , the majority of spin states are fully occupied. This persists in going to $\mathrm{Co}_{5}$ and unoccupied states are dominated by minority spins as shown in Fig. 1 (left panel). All the five Co atoms contribute to the states around HOMO-LUMO (HL), which are shown in Figure 1.

The free $\mathrm{Co}_{5}$ cluster used in this work has MAE of only $8 \mathrm{~K}$ due to its symmetrical structure. However, recent studies have indicated that the transition metal based nanoparticles where the transition metal layers are separated by carbon (C) layers, exhibit large MAE. ${ }^{31,32}$ We have also shown recently that small Co clusters diluted with $\mathrm{C}$ undergo significant enhancement in MAE. ${ }^{33}$ Such enhancement of MAE in transition metal carbide (TMC) results due to the mixing of $p$ states of $\mathrm{C}$ with $\mathrm{d}$ states of transition metal. In this work, we have adopted a different approach to enhance the MAE of the cluster simply by sandwiching the cluster between two benzene rings $\left(\mathrm{C}_{6} \mathrm{H}_{6}\right)$, as shown in Fig. 1. The binding of $\mathrm{Co}_{5}$ cluster with benzene is quite strong as our calculation shows that the binding energy of the combined system is about $3.4 \mathrm{eV}$. Electronic and magnetic properties of the

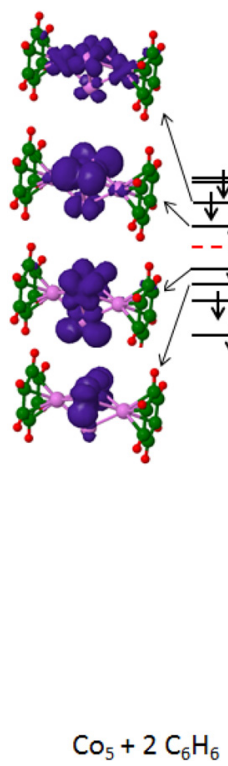

$\mathrm{CO}_{5}$

$\mathrm{Co}_{5}+2 \mathrm{C}_{6} \mathrm{H}_{6}$
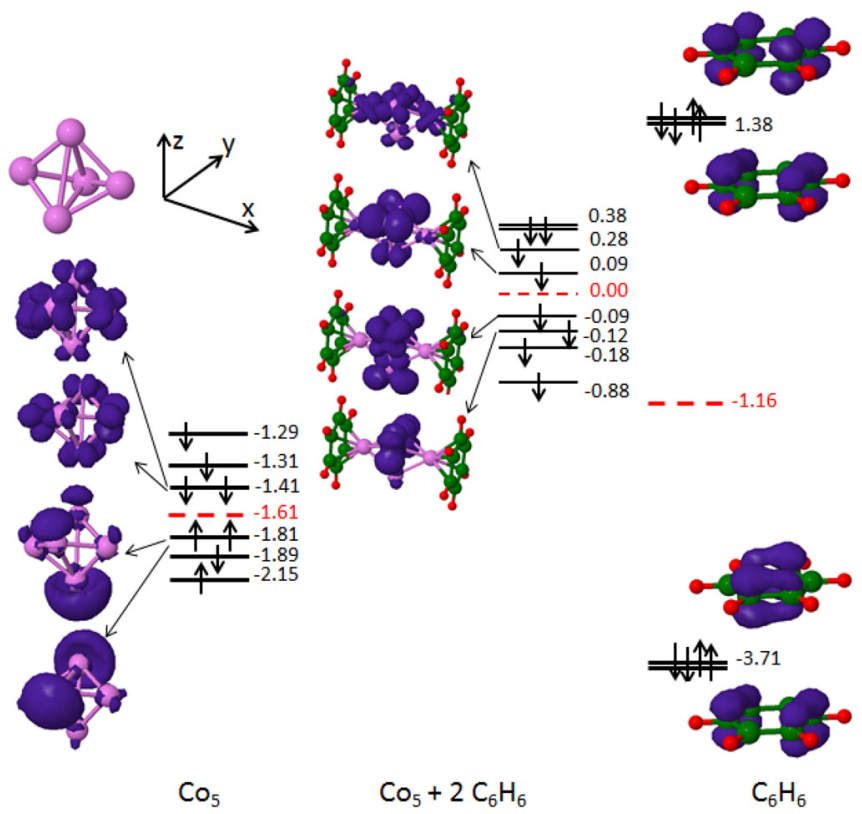

FIG. 1. Single particle states (without SOC) around HOMO and LUMO of $\mathrm{Co}_{5}$ cluster (left), benzene ring (right) and $\mathrm{Co}_{5}$ cluster sandwiched between two benzene. The red dotted line indicates the Fermi level. HOMO and LUMO of each cases are plotted. All the energies are in units of eV.

cluster change significantly due to the bonding with benzene. We note from Fig. 1 that the contribution of the two Co atoms on $\mathrm{C}_{3}$ axis, which are directly linked with benzene, to the states near HL reduces considerably since these two atoms are affected most by the hybridization between the $\mathrm{d}$ states of $\mathrm{Co}_{5}$ cluster and $\pi$ orbitals of benzene. This mixing reduces total magnetic moment from $13 \mu_{B}$ to $7 \mu_{B}$. The local moments of the three Co atoms at the central plane of the cluster reduce slightly from free cluster value of $2 \mu_{B}$ to $\sim 1.9 \mu_{B}$ but the other two Co atoms that are directly linked to benzene suffer large reduction to $0.7 \mu_{B}$. The calculated charge within each atomic sphere of Co atoms is unaffected by benzene, indicating that charge transfer has little effect in these systems. The HL gap also reduces from $0.40 \mathrm{eV}$ to $0.18 \mathrm{eV}$ as shown in Table I. Since the change in GS energy, according to Eqs. (3) and (4), depends on the energy gap between occupied and unoccupied single particle states around HL, the reduction in HL gap, consequently, enhances MAE to about $21 \mathrm{~K}$ with easy magnetization direction along the $\mathrm{C}_{3}$ axis of the cluster.

We now focus on the effects of charging on MAE and magnetization vector of the cluster. When a charge is added or removed from the system, both electronic and magnetic properties change since the single particle states are now modified. Fig. 2 shows that while for a neutral state both

TABLE I. Electronic and magnetic properties of different charged states of $\mathrm{Co}_{5}$ cluster sandwiched between two benzene rings.

\begin{tabular}{lcccc}
\hline \hline System & Charge & HL gap & Spin moment & MAE \\
\hline & state & $(\mathrm{eV})$ & $\left(\mu_{B}\right)$ & $(\mathrm{K})$ \\
$\mathrm{Co}_{5}$ & 0 & 0.40 & 13 & 8.5 \\
$\mathrm{Co}_{5}+$ & -1 & 0.41 & 6 & 17.2 \\
2 benzene & 0 & 0.18 & 7 & 20.6 \\
& +1 & 0.32 & 8 & 53.5 \\
\hline \hline
\end{tabular}



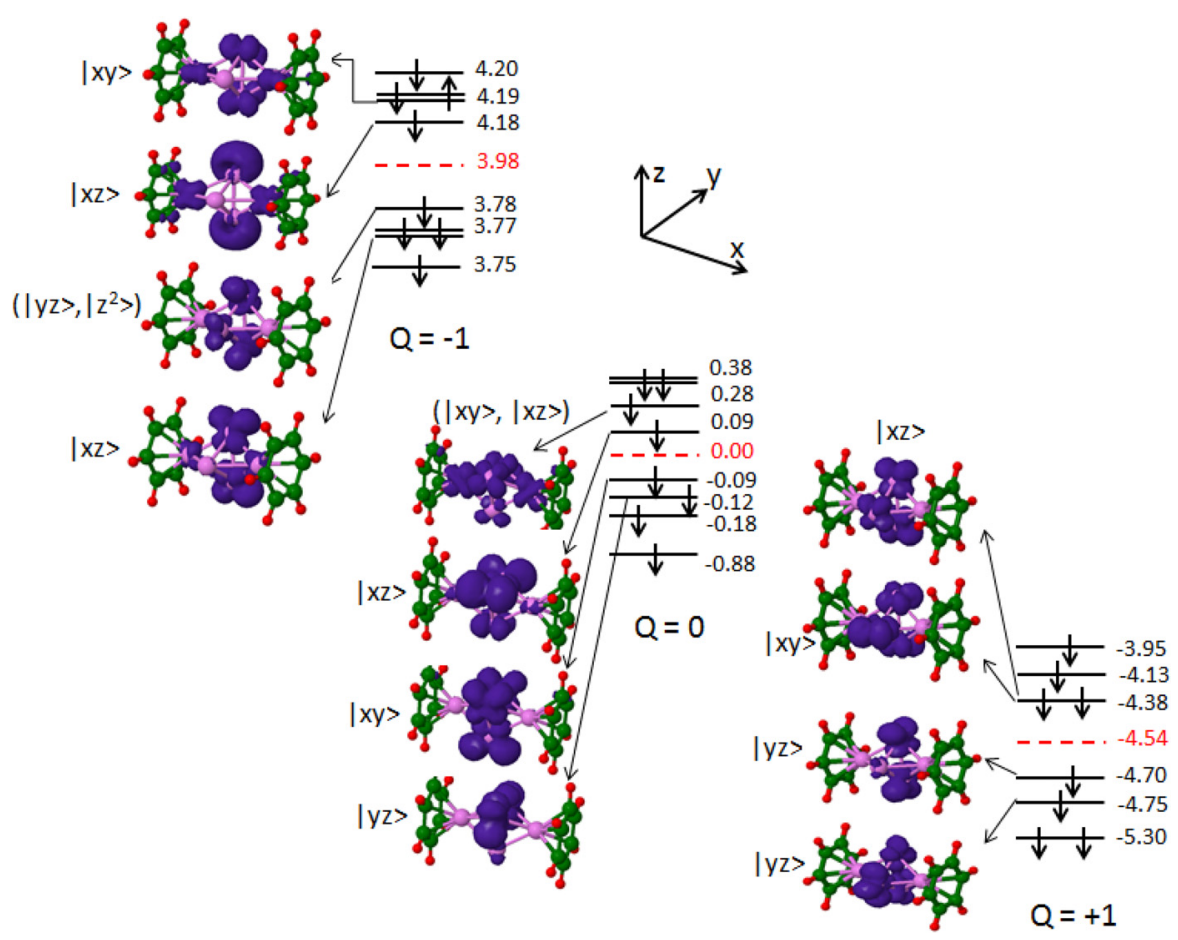

FIG. 2. Single particle states (without SOC) around HOMO and LUMO of $\mathrm{Co}_{5}$ cluster for different charge states which are dominated by minority spins. All the energies are in units of $\mathrm{eV}$. Predominant $\mathrm{d}$ orbital character of two occupied and two unoccupied states are also shown in the figure.

occupied and unoccupied levels around HL are dominated by minority electrons, $\mathrm{Q}=-1$ charge state has a majority spin state above the Fermi level, which contributes to the reduction of SOC energy (Eq. (3)) as well as orbital moment (Eq. (7)). However, for $\mathrm{Q}=+1$ charge state, spin ordering remain unchanged. The HL gap for both charged states are larger compared to neutral state, but for $\mathrm{Q}=-1$ state, the gap is largest. The magnetic moment increases (decreases) by $1 \mu_{B}$ for $\mathrm{Q}=+1(\mathrm{Q}=-1)$ charge state. The calculated ionization energy and electron affinity of the cluster is $4.91 \mathrm{eV}$ and $0.66 \mathrm{eV}$, respectively.

The MAE for $\mathrm{Q}=-1$ charge state is slightly reduced compared to the neutral case, whereas for $\mathrm{Q}=+1$ charge state, MAE is increased by a factor of 2.5. The changes in MAE due to charging of the system can be understood from electronic structure of the respective charge states shown in Fig. 2 in conjunction with Eq. (3). The presence of a majority spin state above the Fermi level in $\mathrm{Q}=-1$ case implies that spin flip term also contributes to the SOC energy but its contribution has opposite sign to that of spin conserving terms. Total magnetic moment also decreases for negative charge states, thus overall energy is reduced. On the other hand, increase in MAE in $\mathrm{Q}=+1$ charge state is primarily due to increase of moment by $1 \mu_{B}$ and reduction in HL gap.

In order to understand the rotation of magnetization direction with charge, we have calculated the orbital moment of the system for each of the three charged states. Since the single particle orbitals are mostly non-degenerate due to crystal field splitting the orbital moment of these states are quenched. However, when SOC is turned on, these nondegenerate states are coupled by spin-orbit Hamiltonian, resulting in non-zero orbital moment. The 1st order change in occupied KS orbitals due to SOC is given by

$$
\left|\phi_{k}, \sigma\right\rangle_{S O}=\lambda \sum_{k^{\prime}} \frac{\left|\phi_{k^{\prime}}, \sigma^{\prime}\right\rangle\left\langle\phi_{k^{\prime}}, \sigma^{\prime}|\mathbf{L} \cdot \mathbf{S}| \phi_{k}, \sigma\right\rangle}{\epsilon_{k}-\epsilon_{k^{\prime}}} .
$$

The total orbital moment is then the sum of the contributions from all occupied orbitals, which can be expressed as

$$
\langle\mathbf{L}\rangle=\lambda \sum_{k k^{\prime}} \frac{\left\langle\phi_{k}, \sigma|\mathbf{L}| \phi_{k^{\prime}}, \sigma^{\prime}\right\rangle\left\langle\phi_{k^{\prime}}, \sigma^{\prime}|\mathbf{L} \cdot \mathbf{S}| \phi_{k}, \sigma\right\rangle}{\epsilon_{k}-\epsilon_{k^{\prime}}} .
$$

Since orbital moment operator, $\mathbf{L}$, does not change the spin, only excited states with the same spin can be reached, i.e., only the 1 st term in Eq. (2) contributes to the orbital moment. Therefore, the projection of orbital moment along a given magnetization direction can be expressed in simplified form as $\mathrm{s}^{30}$

$$
\hat{\mathbf{S}} \cdot \mathbf{L}=-4 \lambda[a(\uparrow, \uparrow)-a(\downarrow, \downarrow)]
$$

We note from Eq. (7) that spin up and spin down electrons have opposite contribution to the moment. Clearly, for Co, which has more than half-filled d electrons, the 2nd term in Eq. (7) will have dominant contribution and according to Eqs. (3) and (7), the easy magnetization axis is along the direction of maximum orbital moment. For the neutral system, calculated orbital moment is largest when magnetization is along $\mathrm{x}$ direction with $\langle L\rangle \approx\left\langle L_{x}\right\rangle=0.53 \mu_{B}$ and MAE calculation by exact diagonalization also confirms that the easy axis is along $x$ direction. For the $Q=-1$ and $Q=+1$ charged states, maximum orbital moments are calculated to be $\langle L\rangle \approx\left\langle L_{y}\right\rangle \approx\left\langle L_{z}\right\rangle=0.47$ and $0.69 \mu_{B}$, respectively (It should be noted that there are several magnetization directions within $10^{\circ}$ of the yz plane that have orbital moment close to that obtained for $y$ and $z$ directions). Therefore, the easy magnetization axis also rotates towards the yz plane, perpendicular to that of the neutral system. A qualitative understanding of this rotation can be obtained from the orbital character of the single particle orbitals of the system. Fig. 2 shows that when an electron is added or removed from the system, orbital character of the states also changes. For instance, while the LUMO of the neutral cluster is 
predominantly of $d_{x y}$ character, the LUMO of $\mathrm{Q}=+1$ state is of $d_{y z}$ character. Consequently, orientation of the orbital moment of the ground state also changes.

A possible experimental set up to detect magnetization rotation in $\mathrm{Co}_{5}$ cluster is shown in Fig. 3. In the proposed setup, the cluster+benzene system is placed between two gold $(\mathrm{Au})$ leads which are connected to a bias voltage. For a neutral system, magnetization points along the symmetry axis of the combined system ( $\mathrm{x}$-axis in this case). When an electron is added or removed from the system via scanning tunneling microscope (STM) tip, magnetization vector rotates by $90^{\circ}$. When spin polarized electrons enter through the lead to the cluster, resistance of the system is larger for the neutral compared to the charged system. Thus, measuring the current, one can detect the rotation of the magnetization vector. In an attempt to examine the stability of the cluster under external contact, we have calculated magnetic properties of the system including leads but without STM tip as shown in Fig. 4. Our calculations show that the magnetic moment of both neutral and charged systems remain unchanged. Interestingly, MAE of the cluster in this case increases further by a factor of 2 to $39 \mathrm{~K}$ for neutral system. For $\mathrm{Q}=-1$ and +1 charge state, calculated MAE are $32 \mathrm{~K}$ and $36 \mathrm{~K}$, respectively. We note from Fig. 4 that HOMO has contributions from $\mathrm{Co}$ as well as benzene and $\mathrm{Au}$ atoms. This mixing results in a stronger orientation of Co $d$ states, and hence increases MAE. Thus, the presence of leads not only preserves magnetic properties but also makes the magnetic properties more robust. However, while for $\mathrm{Q}=+1$ state magnetization rotates by $90^{\circ}$, no rotation is obtained when a charge is added to the system. The reason is that magnetization rotation in the cluster described above crucially depends on the changes in the orbital distributions of electronic states that are primarily localized within the cluster region when a charge is added or removed from the system. While, this may be achieved if the coupling between the leads and the cluster
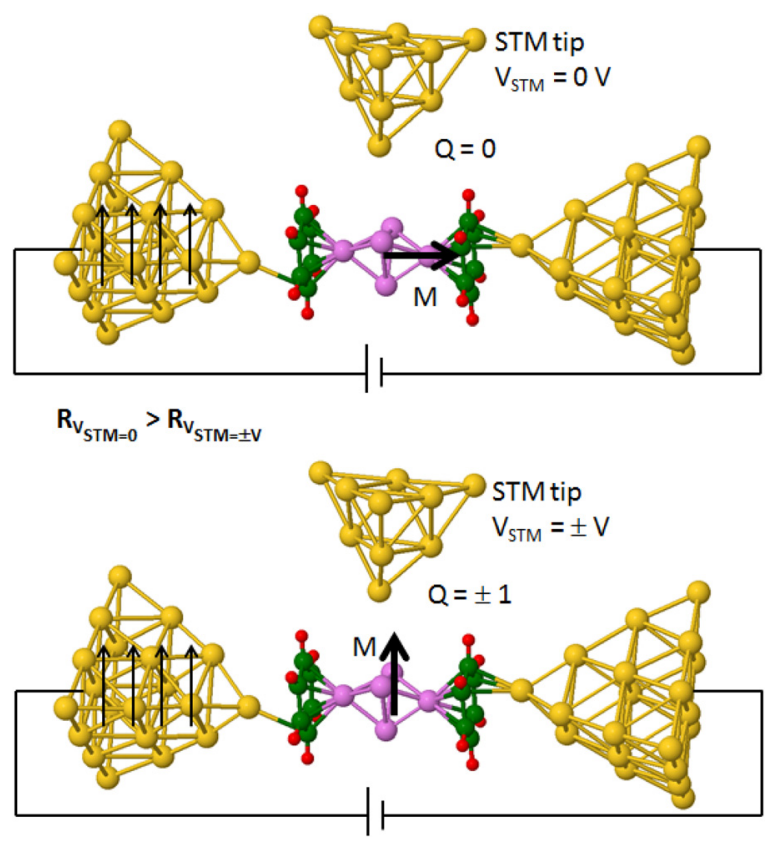

FIG. 3. Schematic of proposed experimental setup for detecting magnetization rotation in $\mathrm{Co}_{5}$ cluster attached to Au lead with STM tip.
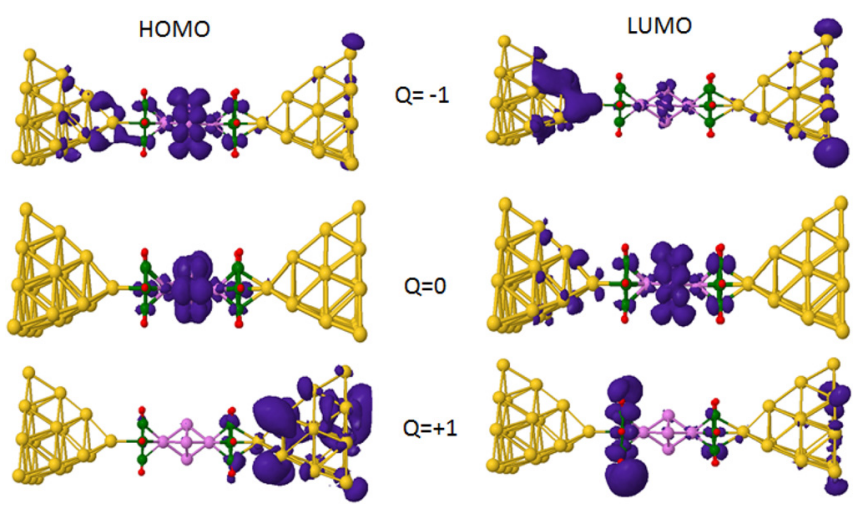

FIG. 4. HOMO and LUMO (without SOC) of different charge states of the $\mathrm{Co}_{5}$ cluster attached to Au leads.

is very weak, but it is difficult to show such effect due to approximate nature of DFT calculations implemented in this work, in addition to the uncertainty associated with finding an appropriate linker between the leads and cluster to make the coupling weak. Since conventional DFT based calculations tend to delocalize electronic states more than they actually are, the state around HL have significant presence at Au leads as shown in Fig. 4 (only HL are shown). So when an electron is added to the system, it spreads though out the system and does not have much effect on orbital distribution of the states localized within the cluster region; hence no rotation. On the other hand, when a particle is removed from the system, orbitals are modified significantly, which leads to the change in orbital moment and consequently, the easy axis. To circumvent this difficulty, special procedure such as constrained DFT is desirable but such method is not implemented in NRLMOL package as of the writing of this paper. Alternatively, one can apply a potential confined within the cluster region of the system. It has been shown that such potential can pull down unoccupied states that are localized within the cluster such that LUMO is dominated by atoms of the cluster. ${ }^{34}$ Therefore, when an electron is added to the system, it can reside within the cluster region, restoring the properties of the system without the leads.

We would like to stress that the rotation of magnetization due to charging is not a general property of any cluster. We have also performed similar calculations with highly symmetric icosahedral $\mathrm{Co}_{13}$ cluster. While anisotropy of the neutral cluster increases to $33 \mathrm{~K}$ when the cluster is sandwiched between two benzene rings, we have not noticed any rotation of magnetization vector when the cluster is charged, suggesting that the rotation of magnetization is a system specific property and one can observe such rotation only in certain class of clusters, possibly with similar symmetry as that of $\mathrm{Co}_{5}$.

In conclusion, we have shown that a $\mathrm{Co}_{5}$ cluster has robust magnetic properties, which are further enhanced when connected to Au leads. This cluster has an exciting property that when a charge is added or removed from the system, its magnetization vector rotates by about $90^{\circ}$, thereby allowing a route to control magnetization even without external electric and magnetic field. Our studies show that small magnetic clusters can be used for designing different spintronic devices to complement more conventional approach based 
on molecules and semiconducting nano-structures. The sandwiched clusters studied in this work can be synthesized experimentally using gas phase techniques, ${ }^{19}$ and their magnetic properties can be investigated using X-ray magnetic circular dichroism, which is recently employed to measure spin coupling and orbital moment along with MAE of free iron clusters. $^{35}$

We gratefully acknowledge support from U.S. Department of Energy (DOE) through Grant No. DE-SC 0006420 for this work.

${ }^{1}$ S. A. Wolf, D. D. Awschalom, R. A. Buhrman, J. M. Daughton, S. von Molnár, M. L. Roukes, A. Y. Chtchelkanova, and D. M. Treger, Science 294, 1488 (2001).

${ }^{2}$ D. Kitchen, A. Richardella, J.-M. Tang, M. E. Flatte, and A. Yazdani, Nat. Mater. 442, 436 (2006).

${ }^{3}$ A. M. Yakunin, A. Y. Silov, P. M. Koenraad, J.-M. Tang, M. E. Flatté, W. V. Roy, J. D. Boeck, and J. H. Wolter, Phys. Rev. Lett. 95, 256402 (2005).

${ }^{4}$ H. B. Heersche, Z. de Groot, J. A. Folk, H. S. J. van der Zant, C. Romeike, M. R. Wegewijs, L. Zobbi, D. Barreca, E. Tondello, and A. Cornia, Phys. Rev. Lett. 96, 206801 (2006).

${ }^{5}$ S. Accorsi, A.-L. Barra, A. Caneschi, G. Chastanet, A. Cornia, A. C. Fabretti, D. Gatteschi, C. Mortal, E. Olivieri, F. Parenti, P. Rosa, R. Sessoli, L. Sorace, W. Wernsdorfer, and L. Zobbi, J. Am. Chem. Soc. 128, 4742 (2006).

${ }^{6}$ L. Bogani and W. Wernsdorfer, Nat. Mater. 7, 179 (2008).

${ }^{7}$ D. Gatteschi, R. Sessoli, and J. Villain, Molecular Nenomagnets (Oxford University Press, Oxford, 2006).

${ }^{8}$ K. Sato, L. Bergqvist, J. Kudrnovský, P. H. Dederichs, O. Eriksson, I. Turek, B. Sanyal, G. Bouzerar, H. Katayama-Yoshida, V. A. Dinh et al., Rev. Mod. Phys. 82, 1633 (2010).

${ }^{9}$ M. Urdampilleta, S. Klyatskaya, J.-P. Cleuziou, M. Ruben, and W. Wernsdorfer, Nat. Mater. 10, 502 (2011).

${ }^{10}$ M. A. AlDamen, S. Cardona-Serra, J. M. Clemente-Juan, E. Coronado, A. Gaita-Ario, C. Mart-Gastaldo, F. Luis, and O. Montero, Inorg. Chem. 48, 3467 (2009).

${ }^{11}$ R. Xiao, D. Fritsch, M. D. Kuz'min, K. Koepernik, H. Eschrig, M. Richter, K. Vietze, and G. Seifert, Phys. Rev. Lett. 103, 187201 (2009).

${ }^{12}$ F. Donati, Q. Dubout, G. Autès, F. Patthey, F. Calleja, P. Gambardella, O. V. Yazyev, and H. Brune, Phys. Rev. Lett. 111, 236801 (2013).
${ }^{13}$ I. G. Rau, S. Baumann, S. Rusponi, F. Donati, S. Stepanow, L. Gragnaniello, J. Dreiser, C. Piamonteze, F. Nolting, and S. O. Gangopadhyay, Science 344, 988 (2014).

${ }^{14}$ D. Awschalom and N. Samarth, Physics 2, 50 (2009).

${ }^{15}$ M. Saito, K. Ishikawa, S. Konno, K. Taniguchi, and T. Arima, Nat. Mater. 8, 634 (2009).

${ }^{16}$ S. Zhang, Y. G. Zhao, P. S. Li, J. J. Yang, S. Rizwan, J. X. Zhang, J. Seidel, T. L. Qu, Y. J. Yang, Z. L. Luo et al., Phys. Rev. Lett. 108, 137203 (2012).

${ }^{17}$ F. A. Cuellar, Y. H. Liu, J. Salafranca, N. Nemes, E. Iborra, G. SanchezSantolino, M. Varela, M. G. Hernandez, J. W. Freeland, M. Zhernenkov et al., Nat. Commun. 5, 4215 (2014).

${ }^{18}$ Y. Mokrousov, N. Atodiresei, G. Bihlmayer, S. Heinze, and S. Blugel, Nanotechnology 18, 495402 (2007).

${ }^{19}$ T. Masubuchi, K. Ohi, T. Iwasa, and A. Nakajima, J. Chem. Phys. 137, 224305 (2012).

${ }^{20}$ S. N. Khanna and S. Linderoth, Phys. Rev. Lett. 67, 742 (1991).

${ }^{21}$ A. J. Cox, J. G. Louderback, and L. A. Bloomfield, Phys. Rev. Lett. 71, 923 (1993).

${ }^{22}$ I. M. Billas, A. Châtelain, and W. A. de Heer, Science 265, 1682 (1994).

${ }^{23}$ J. Kortus, T. Baruah, M. R. Pederson, C. Ashman, and S. N. Khanna, Appl. Phys. Lett. 80, 4193 (2002).

${ }^{24}$ X. Xu, S. Yin, R. Moro, and W. A. de Heer, Phys. Rev. Lett. 95, 237209 (2005).

${ }^{25}$ S. A. Claridge, A. W. Castleman, S. N. Khanna, C. B. Murray, A. Sen, and P. S. Weiss, ACS Nano 3, 244 (2009).

${ }^{26}$ M. R. Pederson and K. A. Jackson, Phys. Rev. B 41, 7453 (1990).

${ }^{27}$ K. Jackson and M. R. Pederson, Phys. Rev. B 42, 3276 (1990).

${ }^{28}$ J. P. Perdew, K. Burke, and M. Ernzerhof, Phys. Rev. Lett. 77, 3865 (1996).

${ }^{29}$ M. R. Pederson and S. N. Khanna, Phys. Rev. B 60, 9566 (1999).

${ }^{30}$ G. van der Laan, J. Phys.: Condens. Matter 10, 3239 (1998).

${ }^{31}$ K. J. Carroll, Z. J. Huba, S. R. Spurgeon, M. Qian, S. N. Khanna, D. M. Hudgins, M. L. Taheri, and E. E. Carpenter, Appl. Phys. Lett. 101, 012409 (2012).

${ }^{32}$ A. A. El-Gendy, M. Qian, Z. J. Huba, S. N. Khanna, and E. E. Carpenter, Appl. Phys. Lett. 104, 023111 (2014).

${ }^{33}$ M. F. Islam and S. N. Khanna, J. Phys.: Condens. Matter 26, 125303 (2014).

${ }^{34}$ J. F. Nossa, M. F. Islam, C. M. Canali, and M. R. Pederson, Phys. Rev. B 88, 224423 (2013).

${ }^{35}$ M. Niemeyer, K. Hirsch, V. Zamudio-Bayer, A. Langenberg, M. Vogel, M. Kossick, C. Ebrecht, K. Egashira, A. Terasaki, T. Möller et al., Phys. Rev. Lett. 108, 057201 (2012). 